\title{
Diagnostic Value of Chitinase-3-like Protein 1 and Keratin 5 in Bronchoalveolar Lavage Fluid of Lung Cancer
}

\author{
BIN HU, WANJIAO CHEN, SHIFANG SUN*, YIFENG MAI AND DECAI ZHU \\ Department of Geriatrics Medicine, The Affiliated Hospital of Medical School, Ningbo University, Ningbo 315020, China
}

Hu et al.: Chitinase-3-like Protein 1 and Keratin 5 in Lung Cancer

\begin{abstract}
To investigate the diagnostic value of chitinase-3-like protein 1 and keratin 5 in bronchoalveolar lavage fluid in 88 patients with lung disease. This was a case control study to collect 50 patients with lung cancer (malignant disease group) and 38 patients with benign lung disease (benign disease group) who were admitted to the respiratory medicine department in the affiliated hospital of medical school of Ningbo university from December 2019 to March 2020. The enzyme-linked immunosorbent assay was applied to detect the expression levels of chitinase-3-like protein 1 and keratin 5 in bronchoalveolar lavage fluid of the two groups of the patients. The diagnostic effect was evaluated using the subject's working characteristics to establish a diagnostic mathematical model. The levels of chitinase-3-like protein 1 and keratin 5 in bronchoalveolar lavage fluid were significantly higher in the malignant disease group than in the benign disease group $(p<0.05)$. These two (chitinase-3-like protein 1 and keratin 5) were significantly correlated and positively correlated and the combined test could significantly improve the sensitivity and specificity of lung cancer diagnosis. The diagnostic rate of chitinase-3-like protein 1 and keratin 5 for lung cancer. The chitinase-3-like protein 1 in bronchoalveolar lavage fluid and carcinoembryonic antigen in blood combined was superior to chitinase-3like protein 1 or keratin 5 alone in the diagnosis of lung cancer. Chitinase-3-like protein 1 and keratin 5 in bronchoalveolar lavage fluid contribute to the early diagnosis of lung cancer and can be used as biomarkers for the diagnosis of lung cancer with high sensitivity and specificity. The application of the two combined assays as molecular markers for clinical diagnosis and as targeted therapeutic agents needs to be further studied.
\end{abstract}

Key words: Lung cancer, bronchoalveolar lavage fluid, chitinase-3-like protein 1, keratin 5

Lung cancer is the leading cause of cancer related deaths worldwide and currently about a quarter of all cancer related deaths are caused by lung cancer, as early lung cancer lacks typical symptoms and about $70 \%$ of patients have distant metastases at the time of diagnosis ${ }^{[1,2]}$. In China, lung cancer is still the most common cancer and how to reduce the cancer burden is the focus of the government system ${ }^{[3]}$ and screening of high risk groups for lung cancer has become the norm. According to studies, the incidence and mortality trends of lung cancer are influenced by population based lung cancer screening programs and lung cancer mortality rates are significantly lower in populations that receive routine screening with low dose Computed Tomography $(\mathrm{CT})^{[4]}$ or volumetric $\mathrm{CT}^{[5]}$ than in populations that do not ${ }^{[5,6]}$, which is attributed to the preferential detection and early diagnosis of early lung cancer patients.

Early recognition and diagnosis is a key to lung cancer

*Address for correspondence

E-mail: fysunshifang@nbu.edu.cn diagnosis, treatment and prognosis ${ }^{[7]}$. In recent years, the detection of biomarkers in alveolar lavage fluid or serum is a new method for the early diagnosis of lung cancer, which is promising due to its simplicity, reproducibility and low invasiveness ${ }^{[8]}$. Recent studies have shown that Chitinase-3-like protein 1 (YKL40) and Keratin 5 (KRT5) play an important role in the staging, differential diagnosis and prognosis of lung cancer ${ }^{[9-11]}$, but most of the studies have been conducted on a single tumor marker and there are few reports on the simultaneous detection of the expression levels of both in alveolar lavage fluid. The aim of this study was to explore the value of YKL40 and KRT5 in Bronchoalveolar Lavage Fluid (BALF) for the early diagnosis of lung cancer. They are reported as follows.

\section{MATERIALS AND METHODS}

Patients and samples:

Fifty patients who were admitted to the respiratory 
medicine department of the Affiliated Hospital of Medicine School, Ningbo University and were clearly diagnosed with lung cancer from December 2019 to March 2020 (benign disease group) were selected. There were 41 males and 9 females, aged $43-81 \mathrm{y}$, with a mean age of $61.5 \pm 8.7 \mathrm{y}$, classified by pathological type, including 24 cases of lung squamous cell carcinoma, 15 cases of lung adenocarcinoma and 11 cases of Small Cell Lung Cancer (SCLC). There were 38 patients with benign lung lesions (malignant disease group), 30 males and 8 females, aged $45-79 \mathrm{y}$, with a mean age of $60.0 \pm 8.0 \mathrm{y}$.

The experimental group inclusion criteria includes age greater than $18 \mathrm{y}$; no radiotherapy or chemotherapy before enrollment; consistent with the diagnosis of lung cancer and histopathological confirmation whereas exclusion criteria includes severe disease unable to tolerate endotracheal endoscopy; unconsciousness or inability to cooperate in completing the study; severe coagulopathy; respiratory infectious diseases.

Control group inclusion criteria includes age greater than $18 \mathrm{y}$; benign lung lesions diagnosed by imaging, histopathology and/or relevant treatment whereas exclusion criteria includes patients with malignant tumors; unconscious or uncooperative to complete the study; severe coagulopathy; with respiratory infectious diseases. The study was approved by the Institutional Review Board of Human Research at the Affiliated Hospital of Medicine School, Ningbo University and all subjects signed an informed consent form ${ }^{[12]}$. There were no significant differences $(\mathrm{p}>0.05)$ in sex, age, height and weight between the test and control groups and the data were comparable.

\section{Biomarker assay:}

The BALF specimens were collected in strict accordance with the standards advocated by the Chinese Medical Association ${ }^{[13]}$ and the detailed steps and eligibility criteria were referred to the previous methods of operation of this group ${ }^{[12,14,15]}$. The level of YKL40 and KRT5 were measured with EnzymeLinked Immunoassay (ELISA) (Beckman Coulter, Inc) and all steps were performed in strict accordance with the instructions. Other tumor markers, including Carcinoembryonic Antigen (CEA), Cancer Antigen 125 (CA125), Carbohydrate Antigen (CA 19-9) and NeuronSpecific Enolase (NSE) were tested on commercially available electrochemiluminescence immunoassay kits
(Beckman Coulter, Brea, CA, USA).

\section{Statistical analysis:}

This research team ${ }^{[12,14,15]}$ adopted Statistical Package for the Social Sciences (SPSS) (version 26.0: SPSS, Chicago, IL, USA) statistical software to analyze the data of YKL40 and KRT5. The normal distribution was expressed as $(\mathrm{x} \pm \mathrm{s})$ and the group t-test was used for comparison between the two groups; the non-normal distribution was expressed as M (P25, P75) and the non-parametric rank sum test was used for comparison between the two groups; the $\chi^{2}$ test was used for comparison of the count data. We drew Receiver Operating Characteristic (ROC) curve of the tumor markers for diagnosis of lung cancer and the Area Under the ROC Curve (AUC), sensitivity and specificity were calculated. The two-sided test level $\alpha=0.05$ with $p<0.05$ indicating a statistically significant difference.

\section{RESULTS AND DISCUSSION}

Table 1 summarizes the basic demographic characteristics for the 88 patients (50 with malignant disease, 38 with benign disease). In the malignant disease group, the mean age was $(61.50 \pm 8.7)$ y and 41 $(82.0 \%)$ were men. In the benign disease group, the mean age (30 men, 8 women) was $(60.00 \pm 8.0)$ y. The number of smokers was $38(76.0 \%)$ in the malignant disease group and $25(65.7 \%)$ in the benign disease group. The malignant disease group included 23 (46.0 $\%$ ) cases of Squamous Cell Carcinoma (SCC), 15 (30.0 $\%$ ) of Adenocarcinoma (ADC) and 12 (24.0\%) of SCLC.

We compared YKL40 and KRT5 level in the patient group with malignant disease and patient group with benign disease. The mean levels of YKL40 was (7135.9 \pm 3174.8$) \mathrm{pg} / \mathrm{ml}$ in the malignant disease group, all significantly higher in patients with benign diseases [(3620.8 \pm 2684.1$) \mathrm{pg} / \mathrm{ml}, \mathrm{p}<0.001]$. The mean levels of KRT5 was $(81.2 \pm 52.6) \mathrm{pg} / \mathrm{ml}$ in the malignant disease group, also significantly higher than that in patients with benign diseases $[(33.2 \pm 39.6) \mathrm{pg} / \mathrm{ml}, \mathrm{p}<0.001]$ (Table 2).

We plotted ROC curves based on the ELISA results to determine the diagnostic effectiveness of the YKL40 and KRT5 levels for lung cancer and included the existing clinical biomarkers, CEA, CA125, CA199 and NSE for comparison (fig. 1 and fig. 2). 
www.ijpsonline.com

TABLE 1: CHARACTERISTICS OF THE STUDY PATIENTS

\begin{tabular}{|c|c|c|}
\hline \multirow{2}{*}{ Characteristic } & \multicolumn{2}{|c|}{ Patient group } \\
\hline & Malignant disease & Benign disease \\
\hline Patients (n) & 50 & 38 \\
\hline Mean age (years) & $61.50 \pm 8.7$ & $60.00 \pm 8.0$ \\
\hline \multicolumn{3}{|l|}{ Sex } \\
\hline Male/Female & $41 / 9$ & $30 / 8$ \\
\hline No. of smokers $(n)$ & 38 & 25 \\
\hline \multicolumn{3}{|l|}{ Histologic type (n) } \\
\hline SCC & & \\
\hline $\mathrm{ADC}$ & & \\
\hline SCLC & & \\
\hline
\end{tabular}

TABLE 2: THE LEVEL OF YKL40, KRT5 IN TWO GROUPS WERE COMPARED (pg/ml, x \pm s)

\begin{tabular}{lccc}
\hline Group & Number & YKL40 & KRT5 \\
\hline Mean malignant disease & 50 & $7135.9 \pm 3174.8$ & $81.2 \pm 52.6$ \\
Mean benign disease & 38 & $3620.8 \pm 2684.1$ & $33.2 \pm 39.6$ \\
$t$ value & & 5.493 & 4.698 \\
p value & & $<0.001$ & $<0.001$ \\
\hline
\end{tabular}
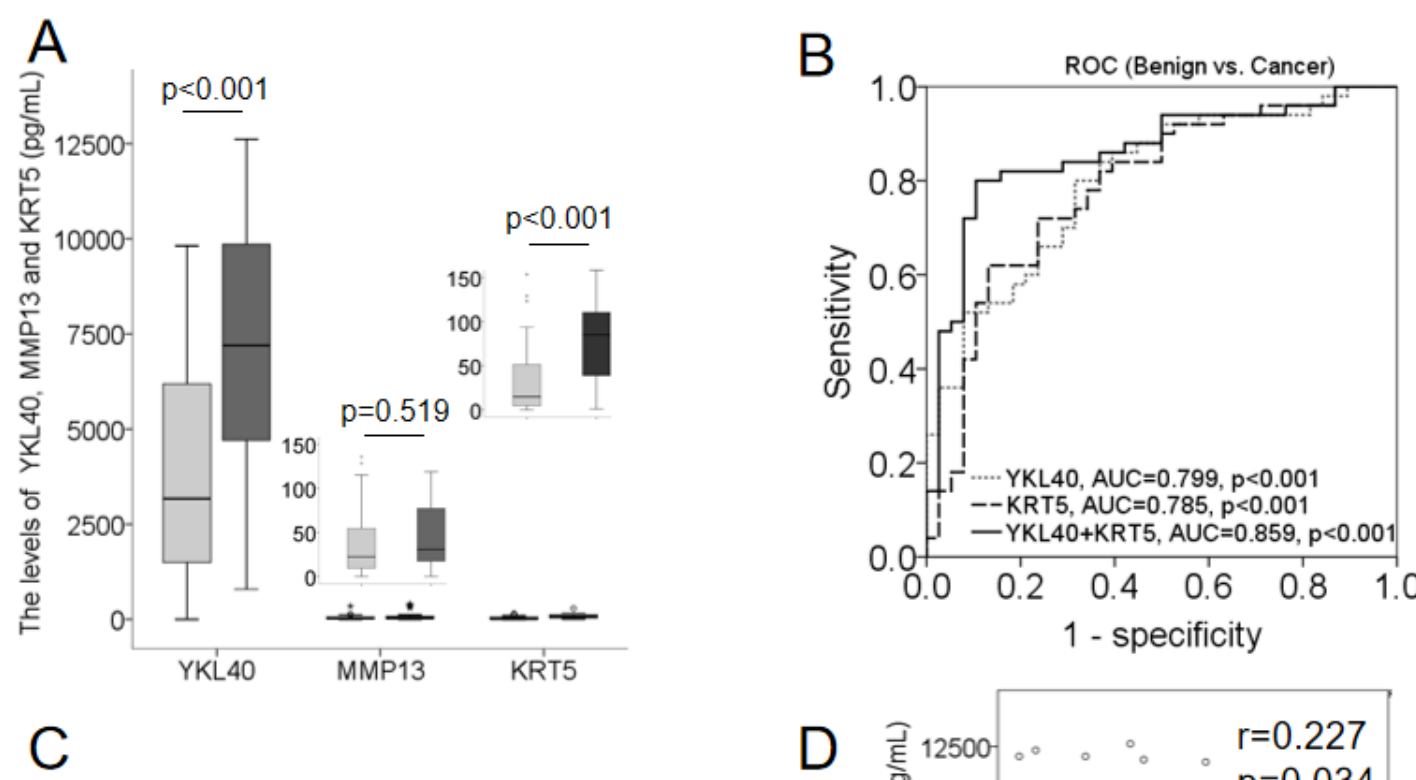

\begin{tabular}{lll}
\hline Benign vs. cancer & Sensitivity & Specificity \\
\hline YKL40 & 0.800 & 0.684 \\
KRT5 & 0.620 & 0.868 \\
YKL40+KRT5 & 0.800 & 0.895 \\
\hline
\end{tabular}

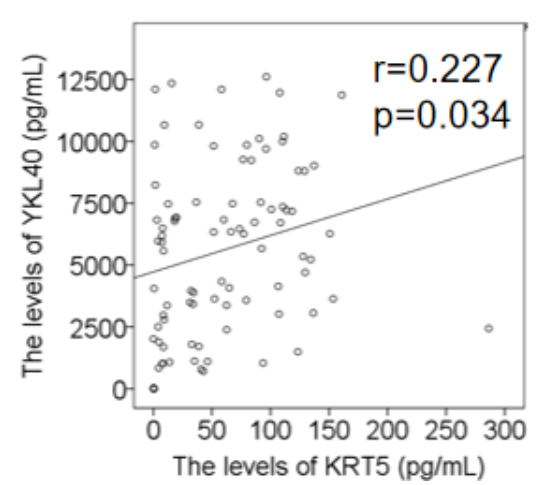

Fig. 1: Comparison of the levels of YKL-40 and KRT5 in bronchoalveolar fluid, (A) Compared with the benign disease group, the levels of YKL-40 and KRT5 in BALF of the malignant disease group were significantly increased, $p<0.001$; (B) ROC curve analysis of YKL40, KRT5 and biomarker combination in differentiating early stage lung cancer patients and benign disease group; (C) The combined detection of YKL-40 and KRT5 can significantly improve the sensitivity of lung cancer diagnosis and specificity; (D) Significant correlation between YKL-40 and KRT5, p=0.034 (p<0.05) and they were positive correlations, $(\square)$ Benign; $(\square)$ Lung cancer 

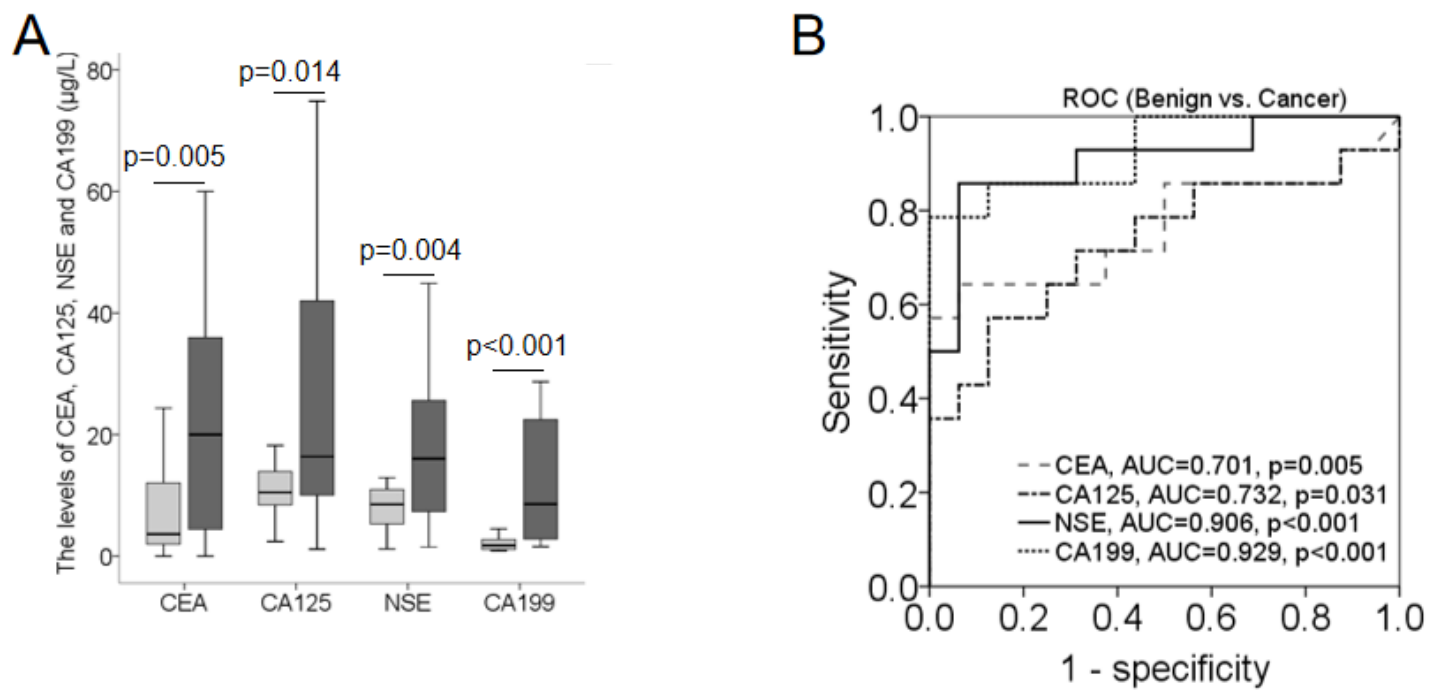

Fig. 2: Blood levels of CEA, CA125, NSE and CA199, (A) Blood levels of CEA, CA125, NSE and CA199 all have some diagnostic value for lung cancer, with CA199 having the greatest significance and its diagnostic value for lung cancer also having the greatest significance for NSE and CA199; (B) The ROC curve showed that the AUC of both was 0.906 and 0.929 respectively with $p<0.001$, ( $\square$ ) Benign; ( $\square$ ) Lung cancer

Fig. 1A showed that compared with the benign disease group, the level of YKL-40 in the malignant disease group was significantly increased, $\mathrm{p}<0.001$ and the difference was statistically significant; compared with the benign disease group, the level of KRT5 in the malignant disease group was significantly increased, $\mathrm{p}<0.001$ and the difference was statistically significant.

Fig. 1B showed that according to the ROC curve, both YKL-40 and KRT5 have diagnostic value for lung cancer. The AUC of YKL-40 alone was 0.799 , while that of KRT5 alone was 0.785 . It was worth noting that the AUC of YKL-40 and KRT5 in combination were 0.859 , which was more significant than that of YKL-40 or KRT5 alone.

Fig. 1C demonstrated that the sensitivity of YKL-40 alone was $80.0 \%$ with a specificity of $68.4 \%$; the sensitivity of KRT5 alone is $62.0 \%$ with a specificity of $86.8 \%$; the sensitivity of YKL-40 and KRT5 is 80.0 $\%$ with a specificity of $89.5 \%$; the AUC of both was 0.859 , which was more significant than that of YKL40 or KRT5 alone. KRT5 could significantly improve the sensitivity and specificity in the diagnosis of lung cancer.

Fig. 1D demonstrated that the correlation study between YKL-40 and KRT5 showed that $\mathrm{p}=0.034(\mathrm{p}<0.05)$, suggesting that there was a significant correlation between the two and they were positive correlations, which means that the two were elevated in patients with lung cancer, but not in the benign disease group.

We also studied and analyzed the levels of CEA, CA125, NSE and CA199 in the blood of patients that could be extracted and obtained the following results: The levels of CEA, CA125, NSE and CA199 in blood have certain diagnostic value for lung cancer, with CA199 having the greatest significance and its diagnostic value for lung cancer is also determined by NSE and CA199. CA199 was the most significant (fig. 2A) and the ROC curves shows that the AUCs of both were 0.906 and 0.929 respectively, with $\mathrm{p}<0.001$, as detailed in fig. $2 \mathrm{~B}$.

It is well known that CEA has the greatest diagnostic value for lung cancer, especially for non-SCLC such as adenocarcinoma and we made a detailed analysis of the diagnostic value of CEA in blood, YKL-40 and KRT5 in BALF for lung cancer. The correlation analysis of YKL-40 suggests that $\mathrm{p}=0.048$ and $\mathrm{r}=0.242$, as detailed in fig. 3A and fig. 3B.

The ROC curves in fig. 4 shows that the combined detection of YKL-40 in BALF and CEA in blood had the greatest significance, resulting in an AUC of 0.824 , sensitivity of $83.8 \%$ and specificity of $76.7 \%$ for the diagnosis of lung cancer, while the combined detection of KRT5 in BALF and CEA in blood have an AUC of 0.777 , sensitivity of $75.7 \%$ and specificity of $76.7 \%$ for the diagnosis of lung cancer. The AUC of serum CEA alone for the diagnosis of lung cancer is 0.701 , with a sensitivity of $62.2 \%$ and a specificity of $80.0 \%$. 

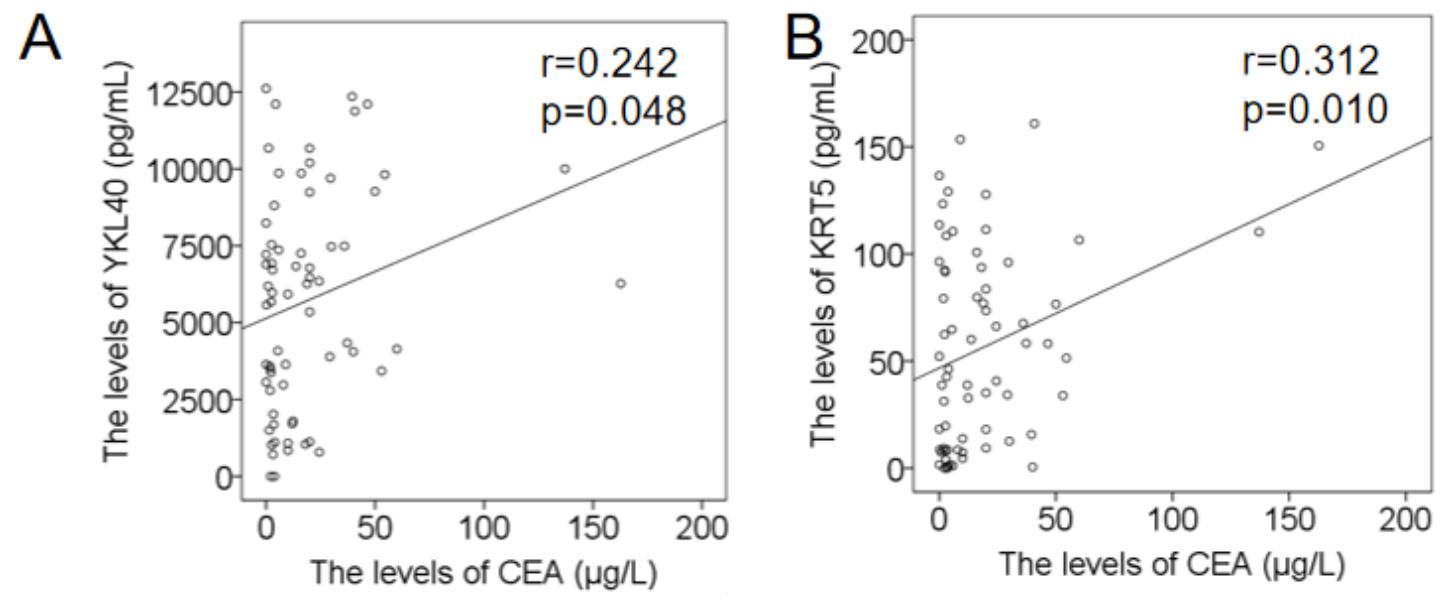

Fig. 3: KRT5 in blood CEA and BALF, (A) KRT5 in blood CEA and BALF had a better correlation, $p=0.010$ and $r=0.312$; (B) While the correlation analysis of YKL-40 in BALF and CEA in blood suggested that $p=0.048$ and $r=0.242$

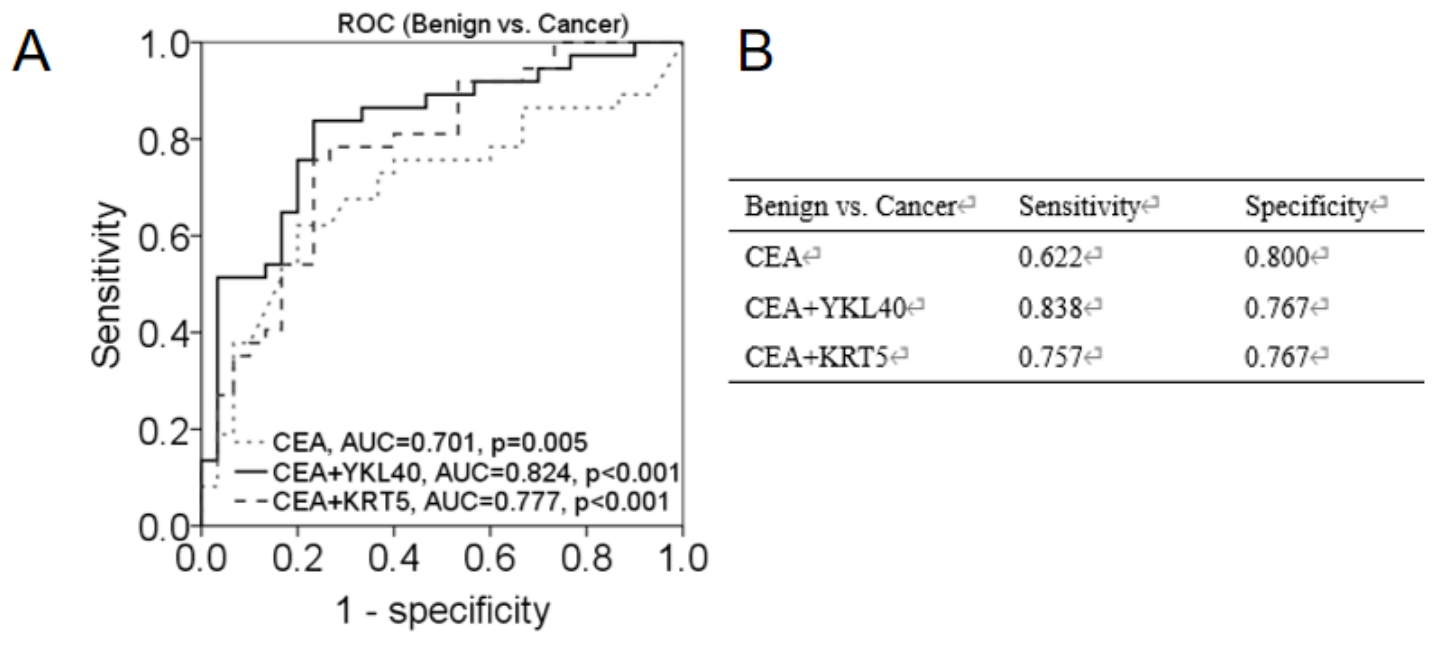

Fig. 4: ROC curves, (A) ROC curves showed that the combined detection of YKL-40 in blood CEA and BALF had the greatest significance, resulting in an AUC of 0.824 sensitivity of $83.8 \%$ and specificity of $76.7 \%$ for the diagnosis of lung cancer; (B) While the combined detection of KRT5 in blood CEA and BALF had an AUC of 0.777 sensitivity of $75.7 \%$ and specificity of $76.7 \%$ for the diagnosis of lung cancer. The AUC of serum CEA alone for the diagnosis of lung cancer was 0.701 , with a sensitivity of $62.2 \%$ and a specificity of $80.0 \%$

Because of no extremely specific for malignant tumors, tumor markers require combined testing, which are mainly used for tumor diagnosis, staging, monitoring of treatment effects and metastasis and recurrence ${ }^{[16-18]}$. In recent years, many studies have reported ${ }^{[12,19,20]}$ that the combination of tumor markers in BALF have significant effects in the diagnosis of lung cancer and are of great clinical significance. In the present study, we added the value of YKL40 and KRT5 in BALF on lung cancer diagnosis and found that the expression levels of YKL40 and KRT5 in BALF of lung cancer patients was significantly increased and the combination of the two was found to be of great significance in the diagnosis of lung cancer. The findings of this study provide some theoretical basis and experimental data for later studies and promote and expand the effective detection methods for early tumor diagnosis. YKL40 is secreted by chondrocytes and macrophages and is mainly associated with the development of inflammatory diseases (e.g., bronchial asthma, nasalpolyps, etc.) and can be used as an inflammatory biomarker ${ }^{[21,22]}$. YKL40 has also been found to be associated with a high risk of cancer progression and future cancer development ${ }^{[9,10,23]}$ KRT5 is widely expressed in cancer tissues and can promote cancer cell growth, migration ${ }^{[11,24]}$ and increase mortality in cancer patients. Xiao et al. ${ }^{[25]}$ found KRT5 to be valuable in the diagnostic differential of lung adenocarcinoma and lung squamous cell carcinoma. Highest are potential biomarkers. Thus, both have the potential to be developed as tumor markers. 
Previous experiments tested the correlation between YKL-40 and KRT5 in BALF and CEA, CA125, NSE and CA199 in peripheral blood. And usually, cytokines in the peripheral blood of lung cancer patients are expressed in parallel with those in BALF or cytokines are elevated earlier in BALF. In summary, if YKL40 and KRT5 in peripheral blood are synchronously expressed with YKL-40 and KRT5 in BALF, then measuring the levels of YKL-40 and KRT5 in BALF is relatively of the greatest significance for the diagnosis of lung cancer. SCLC after evaluation and therefore further experimental validation in large samples is required. This study intends to evaluate the correlation between the expression of BALF and blood YKL-40 and KRT5 and tumor markers in large samples with lung cancer stage and pathological type to determine their significance and mechanism of action in lung cancer invasion and metastasis, with a view to studying their application as molecular markers for clinical diagnosis and targeted therapeutic agents.

\section{Authors' contributions:}

Shifang Sun, Yifeng Mai and Decai Zhu were considered as co-corresponding authors.

\section{Acknowledgements:}

This work was supported by the by the Natural Science Foundation of Ningbo (Grant No. 2018A610205) and the Natural Science Foundation of Ningbo (Grant No. 2016C51019, 2019C50080).

\section{Conflict of interests:}

The authors report no conflicts of interest.

\section{REFERENCES}

1. Siegel RL, Miller KD, Jemal A. Cancer statistics, 2020. CA Cancer J Clin 2020;70(1):7-30.

2. de Martel C, Georges D, Bray F, Ferlay J, Clifford GM. Global burden of cancer attributable to infections in 2018: A worldwide incidence analysis. Lancet Glob Health 2020;8(2):e180-90.

3. Cao M, Li H, Sun D, Chen W. Cancer burden of major cancers in China: A need for sustainable actions. Cancer Commun 2020;40(5):205-10.

4. Becker N, Motsch E, Trotter A, Heussel CP, Dienemann H, Schnabel PA, et al. Lung cancer mortality reduction by LDCT screening-Results from the randomized German LUSI trial. Int J Cancer 2020;146(6):1503-13.

5. de Koning HJ, van der Aalst CM, de Jong PA, Scholten ET, Nackaerts K, Heuvelmans MA, et al. Reduced lung-cancer mortality with volume CT screening in a randomized trial. N Engl J Med 2020;382(6):503-13.

6. Yang D, Liu Y, Bai C, Wang X, Powell CA. Epidemiology of lung cancer and lung cancer screening programs in China and the United States. Cancer Lett 2020;468:82-7.
7. Li T, Liu Y, Zhang W, Lin L, Zhang J, Xiong Y, et al. A rapid liquid biopsy of lung cancer by separation and detection of exfoliated tumor cells from bronchoalveolar lavage fluid with a dual-layer "PERFECT" filter system. Theranostics 2020;10(14):6517-29.

8. Rijavec E, Coco S, Genova C, Rossi G, Longo L, Grossi F. Liquid biopsy in non-small cell lung cancer: Highlights and challenges. Cancers 2020;12(1):17.

9. Park KR, Yun HM, Yoo K, Ham YW, Han SB, Hong JT. Chitinase 3 like 1 suppresses the stability and activity of p53 to promote lung tumorigenesis. Cell Commun Signal 2020;18(1):1-3.

10. Bian B, Li L, Yang J, Liu Y, Xie G, Zheng Y, et al. Prognostic value of YKL-40 in solid tumors: A meta-analysis of 41 cohort studies. Cancer Cell Int 2019;19(1):1-5.

11. Zhang Z, Tu K, Liu F, Liang M, Yu K, Wang Y, et al. FoxM1 promotes the migration of ovarian cancer cell through KRT5 and KRT7. Gene 2020;757:144947.

12. Sun SF, Chen ZB, Zhang Y. Diagnostic value of CA724 and CA199 in bronchoalveolar lavage fluid of lung cancer. Chin Gen Pract 2018;21(6):653-7.

13. Chinese MedicalAssociation of Respiratory Diseases. Technical specification for cytological detection of bronchoalveolar lavage fluid. Chin J Tuberc Respir Dis 2002;25(7):390-1.

14. Cao C, Chen ZB, Sun SF, Yu YM, Ding QL, Deng ZC. Evaluation of VEGF-C and tumor markers in bronchoalveolar lavage fluid for lung cancer diagnosis. Sci Rep 2013;3(1):1-4.

15. Cao C, Sun SF, Lv D, Chen ZB, Ding QL, Deng ZC. Utility of VEGF and sVEGFR-1 in bronchoalveolar lavage fluid for differential diagnosis of primary lung cancer. Asian Pac J Cancer Prev 2013;14(4):2443-6.

16. Liu L, Teng J, Zhang L, Cong P, Yao Y, Sun G, et al. The combination of the tumor markers suggests the histological diagnosis of lung cancer. Biomed Res Int 2017;2017:2013989.

17. Ren X, Zhang Y, Lyu Y, Jin B, Guo H, Wu J, et al. Lactate dehydrogenase and serum tumor markers for predicting metastatic status in geriatric patients with lung adenocarcinoma. Cancer Biomark 2019;26(2):139-50.

18. Faria SC, Sagebiel T, Patnana M, Cox V, Viswanathan C, Lall $\mathrm{C}$, et al. Tumor markers: Myths and facts unfolded. Abdom Radiol 2019;44(4):1575-600.

19. Li Y, Tian X, Gao L, Jiang X, Fu R, Zhang T, et al. Clinical significance of circulating tumor cells and tumor markers in the diagnosis of lung cancer. Cancer Med 2019;8(8):3782-92.

20. Korkmaz ET, Koksal D, Aksu F, Dikmen ZG, Icen D, Maden $\mathrm{E}$, et al. Triple test with tumor markers CYFRA 21.1, HE4 and ProGRP might contribute to diagnosis and subtyping of lung cancer. Clin Biochem 2018;58:15-9.

21. Ma Y, Zheng C, Shi L. The role of YKL40 in the pathogenesis of CRS with nasal polyps. Eur Arch Otorhinolaryngol 2018;275(2):431-8.

22. El Basha NR, Osman HM, Abdelaal AA, Saed SM, Shaaban HH. Increased expression of serum periostin and YKL40 in children with severe asthma and asthma exacerbation. J Invest Med 2018;66(8):1102-8.

23. Kjaergaard AD, Nordestgaard BG, Johansen JS, Bojesen SE. Observational and genetic plasma YKL-40 and cancer in 96 099 individuals from the general population. Int J Cancer 2015;137(11):2696-704.

24. Fujiwara S, Deguchi S, Magin TM. Disease-associated keratin mutations reduce traction forces and compromise adhesion and collective migration. J Cell Sci 2020;133(14):jcs243956. 
25. Xiao J, Lu X, Chen X, Zou Y, Liu A, Li W, et al. Eight potential biomarkers for distinguishing between lung adenocarcinoma and squamous cell carcinoma. Oncotarget 2017;8(42):71759-71.
This is an open access article distributed under the terms of the Creative Commons Attribution-NonCommercial-ShareAlike 3.0 License, which allows others to remix, tweak, and build upon the work non-commercially, as long as the author is credited and the new creations are licensed under the identical terms

This article was originally published in a special issue, "Novel Therapeutic Approaches in Biomedicine and Pharmaceutical Sciences" Indian J Pharm Sci 2021:83(6) Spl Issue "236-242" 